\title{
Efficient Use of Closed-Form Green's Functions for the Analysis of Planar Geometries with Vertical Connections
}

\author{
Noyan Kınayman, Member, IEEE, and M. I. Aksun, Member, IEEE
}

\begin{abstract}
An efficient and rigorous method for the analysis of planarly layered geometries with vertical metallizations is presented. The method is based on the use of the closed-form spatial-domain Green's functions in conjunction with the method of moments (MoM). It has already been demonstrated that the introduction of the closed-form Green's functions into the MoM formulation results in significant computational improvement for the analysis of planar geometries. However, in cases of vertical metallizations, such as shorting pin's, via holes, etc., there are some difficulties in incorporating the closed-form Green's functions into the MoM formulation. In this paper, these difficulties are discussed and their remedies are proposed. The proposed approach is compared to traditional approaches from a theoretical point of view, and the numerical implementation is demonstrated through some examples. The results are also compared to those obtained from the commercial software $\mathrm{em}$ by SONNET.
\end{abstract}

Index Terms-Closed-form Green's functions, generalized pencil of function method, method of moments, planarly layered media.

\section{INTRODUCTION}

$\mathbf{R}$ ECENT advances in monolithic microwave integrated circuits (MMIC's) and ever-increasing speed of digital circuits have prompted the need for a rigorous and efficient electromagnetic (EM) modeling technique. Therefore, there have been a flurry of activities in the area of computational electromagnetics to develop computationally efficient and accurate numerical techniques for modeling and simulating the electrical performances of such circuits. There are basically three numerical techniques, used commonly for EM simulations, that have attracted considerable interest; namely the method of moments (MoM) and its variants [1], the finite-element method (FEM) [2], and the finite-difference time-domain (FDTD) method [3]. Among these approaches, the spatial-domain MoM is widely regarded as one of the most popular techniques for the solution of the mixed-potential integral equation (MPIE) for printed geometries in planarly layered media [4], [5].

Formulation of the spatial-domain MoM for the analysis of printed geometries begins with writing the MPIE in terms

Manuscript received May 6, 1996; revised January 24, 1997. This work was supported in part by NATO's Scientific Affairs Division in the framework of the Science for Stability Program and in part by the Scientific and Technical Research Council of Turkey (TUBITAK) under Contract EEEAG-132.

The authors are with the Department of Electrical and Electronics Engineering, Bilkent University, 06533 Ankara, Turkey.

Publisher Item Identifier S 0018-9480(97)02896-2. of the Green's functions of vector and scalar potentials in a multilayer medium. Then, the integral equation is discretized by expanding the unknown current densities in terms of known basis functions and by applying the boundary conditions in integral sense through the testing procedure of the MoM. This formulation has the advantage of employing the MPIE, whose kernel shows a weak surface integrable singularity while the electric-field integral equation involves stronger singularity, but it requires the Green's functions in the spatial domain. The spatial-domain Green's functions are obtained from the spectral-domain Green's functions via the Hankel transformation, in which the spectral-domain Green's functions are known in closed forms for layered media [6], [7]. This transformation, also known as the Sommerfeld integral, contains oscillatory integrand over an infinite domain whose evaluation is computationally very expensive; hence, the apparent disadvantage of the spatial-domain MoM.

It was recently demonstrated that the computational burden in the calculation of the spatial-domain Green's functions can be circumvented by approximating the spectral-domain Green's functions in terms of complex exponentials whose Hankel transforms can be analytically obtained via the Sommerfeld identity [8]. Hence, the spatial-domain Green's functions for vector and scalar potentials can be cast into so-called closed forms which are finite sums of complex images. In this approach, the crucial step is the numerical implementation of exponential approximation, which can be performed by using Prony's techniques [9] or techniques based on the pencil of functions [10], [11]. The original derivation of the closed-form Green's functions, as proposed in [8], employed the original Prony method and was limited in use to thick and single layer structures, which was due to inadequacy of the original Prony method. This problem was eliminated by employing the least squares Prony method [12], and then the approximation was further improved by using the generalized pencil-of-functions method (GPOF) [7], which is less noise sensitive and more robust as compared to the Prony methods. However, the algorithm for the exponential approximation was still computationally expensive, because Prony's methods and the GPOF method require uniform sampling of the function to be approximated along the range of approximation. This, in turn, makes it necessary to take a large number of samples for functions with local oscillations and fast variations, like spectral-domain Green's functions in general, rendering the algorithm computationally expensive and not robust. Recently, 
a two-level approach that requires piecewise uniform sampling has been introduced to eliminate this problem, and is demonstrated to be much more efficient and robust [13]. Hence, the spatial-domain closed-form Green's functions can be employed efficiently in the solution of MPIE for planar, multilayer geometries.

It has already been demonstrated that the use of the closedform Green's functions in the solution of the MPIE via the MoM improves the computational efficiency of the method, even before the introduction of the robust, two-level approach [14]-[17]. In addition, it has recently been shown that MoM matrix entries can be evaluated analytically if the closedform Green's functions are employed [18]. Consequently, the computational efficiency of the spatial-domain MoM is further improved, making this approach a powerful candidate for a general computer-aided design (CAD) software. But, it should be noted that all the cases studied so far, with the use of the closed-form Green's functions, consist of only horizontal planar conductors, except for a few cases in which some simplifying assumptions, such as electrically short vertical metallizations and only vertical metallizations in a one- or two-layer medium, have been imposed [19], [20]. Further investigation has shown that the improvement in the computational efficiency may not be achieved for three-dimensional (3-D) geometries in planarly layered media, simply by casting the spatial-domain Green's functions into closed forms. In this paper, the authors discuss the difficulties involved in using the spatial-domain closed-form Green's functions in conjunction with the MoM for 3-D multilayer geometries, and propose and demonstrate an efficient technique to improve the computational efficiency of the MoM for such geometries.

Since this paper addresses the difficulties of the use of the closed-form Green's functions in the MoM for 3-D planar geometries, it would be instructive to present the spatial-domain MoM formulation, and to briefly discuss the derivation of the closed-form Green's functions in Section II. In Section III, the authors discuss the difficulties involved in this formulation, and some remedies are proposed. Section IV presents some results demonstrating the applications of the formulation to geometries with vertical metallizations and compares these results to those obtained from the commercial software em by SONNET. In Section V, conclusions are given.

\section{Formulation OF THE PROBLEM}

Consider, for the sake of illustration, a general microstrip structure in a multilayer environment, as shown in Fig. 1. It is assumed that all layers and the ground plane extend to infinity in the horizontal plane, and the conductors are lossless and infinitesimally thin. The thickness and the permittivity of $i$ th layer are denoted by $d_{i}$ and $\epsilon_{r_{i}}$, respectively. Although the geometry depicted in Fig. 1 shows only one vertical metallization, the formulation given here applies to multiple vertical metallizations, which is demonstrated with an example in the section of results and discussions. A time convention of $e^{j \omega t}$ has been adopted in this paper.

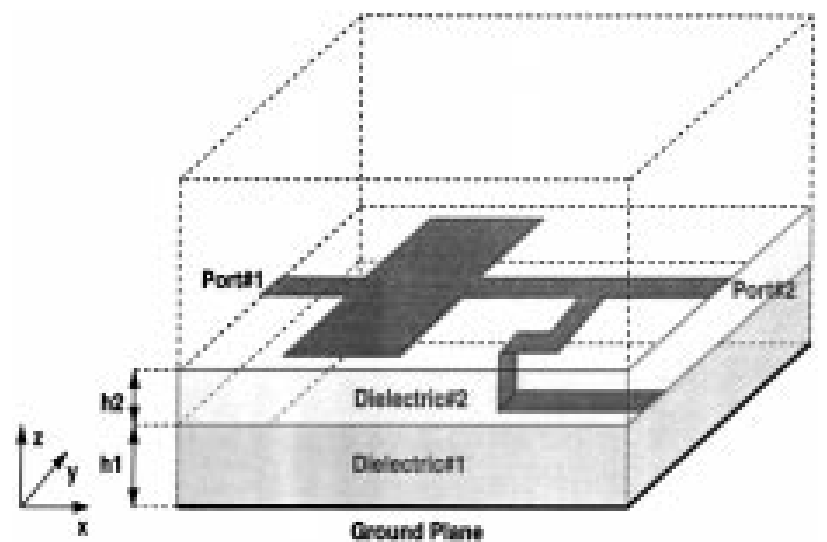

Fig. 1. A typical geometry with a vertical metallization in a multilayer medium.

\section{A. MPIE Formulation and MoM Matrix Entries}

The tangential components of the electric field on the plane of the patch and on the vertical strips can be written in terms of the surface current density J, and the associated Green's functions of vector and scalar potentials as follows:

$$
\begin{aligned}
E_{x}= & -j w G_{x x}^{A} * J_{x}+\frac{1}{j w} \frac{\partial}{\partial x}\left(G^{q_{e}} * \nabla \cdot \mathbf{J}\right) \\
E_{y}= & -j w G_{y y}^{A} * J_{y}+\frac{1}{j w} \frac{\partial}{\partial y}\left(G^{q_{e}} * \nabla \cdot \mathbf{J}\right) \\
E_{z}= & -j w G_{z x}^{A} * J_{x}-j w G_{z y}^{A} * J_{y}-j w G_{z z}^{A} * J_{z} \\
& +\frac{1}{j w} \frac{\partial}{\partial z}\left(G^{q_{e}} * \nabla \cdot \mathbf{J}\right)
\end{aligned}
$$

where $*$ denotes convolution and $G_{x x}^{A}=G_{y y}^{A}$. The term $G_{i j}^{A}$ represents the $i$-directed vector potential at $\mathbf{r}$ due to a $j$-directed electric dipole of unit strength located at $\mathbf{r}^{\prime}$, while $G^{q e}$ represents the scalar potential by a unit point charge associated with an electric dipole. Since the traditional form of the Green's functions are employed in the formulation [21], the Green's function of the scalar potential is not unique for horizontal and vertical electric dipoles. Hence, the term involving the Green's function of the scalar potential, which is common in (1)-(3) can be explicitly written as

$$
G^{q_{e}} * \nabla \cdot \mathbf{J}=G_{x}^{q_{e}} * \frac{\partial J_{x}}{\partial x}+G_{y}^{q_{e}} * \frac{\partial J_{y}}{\partial y}+G_{z}^{q_{e}} * \frac{\partial J_{z}}{\partial z}
$$

where $G_{x}^{q_{e}}\left(=G_{y}^{q_{e}}\right)$ and $G_{z}^{q_{e}}$ denote the Green's functions of the scalar potential for a horizontal and vertical electric dipoles, respectively.

To solve for the surface current density $\mathbf{J}$ via the MoM, J is expressed as a linear combination of the sub-domain basis functions

$$
\begin{aligned}
J_{x}(x, y) & =\sum_{m} \sum_{n} I_{x}^{(m n)} B_{x}^{(m n)}(x, y) \\
J_{y}(x, y) & =\sum_{m} \sum_{n} I_{y}^{(m n)} B_{y}^{(m n)}(x, y) \\
J_{z}(x, y, z) & =\sum_{l} I_{z}^{(l)} B_{z}^{(l)}(x, y, z)
\end{aligned}
$$




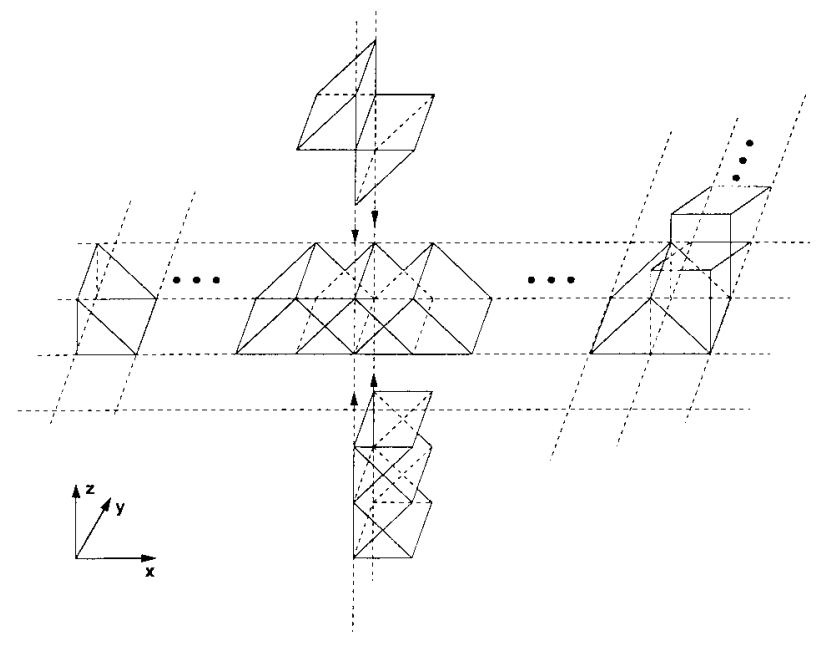

Fig. 2. Basis functions representing the current density.

where $B_{x}^{(m n)}, B_{y}^{(m n)}$, and $B_{z}^{(l)}$ are the basis functions with the unknown coefficients $I_{x}^{(m n)}, I_{y}^{(m n)}$, and $I_{z}^{(l)}$, defined at $(m, n)$ th position on the subdivided horizontal conductor and at $l$ th position on the subdivided vertical conductor. In this paper, rooftop functions are chosen as the basis functions to represent $x-, y$, and $z$-components of the current density, as shown in Fig. 2. The sources are modeled as current filaments, therefore, it is suitable to use half-rooftop basis functions at the source terminals, as well as at the sink terminals where the shorting pins or via holes are terminated in the ground plane. At the intersections of vertical and horizontal conductors, half-rooftop and saw-tooth basis functions are employed on the vertical and horizontal conductors, respectively, whose amplitudes are related to satisfy the conservation of charges, (see Fig. 2).

Following the substitution of (5)-(7) into (1)-(3), the boundary conditions for the tangential electric fields are implemented in integral sense through the testing procedure of the MoM, for which the field expressions multiplied by some testing functions $T_{x}^{\left(m^{\prime} n^{\prime}\right)}, T_{y}^{\left(m^{\prime} n^{\prime}\right)}$, and $T_{z}^{\left(l^{\prime}\right)}$ are integrated on the conductors and are set to zero. This leads to a matrix equation for the unknown coefficients of the basis functions as

$$
\left[\begin{array}{ccc}
Z_{x x}^{\left(m^{\prime} n^{\prime}, m n\right)} & Z_{x y}^{\left(m^{\prime} n^{\prime}, m n\right)} & Z_{x z}^{\left(m^{\prime} n^{\prime}, l\right)} \\
Z_{y x}^{\left(m^{\prime} n^{\prime}, m n\right)} & Z_{y y}^{\left(m^{\prime} n^{\prime}, m n\right)} & Z_{y z}^{\left(m^{\prime} n^{\prime}, l\right)} \\
Z_{z x}^{\left(l^{\prime}, m n\right)} & Z_{z y}^{\left(l^{\prime}, m n\right)} & Z_{z z}^{\left(l^{\prime}, l\right)}
\end{array}\right]\left[\begin{array}{c}
I_{x}^{(m n)} \\
I_{y}^{(m n)} \\
I_{z}^{(l)}
\end{array}\right]=\left[\begin{array}{c}
V_{x}^{\left(m^{\prime} n^{\prime}\right)} \\
V_{y}^{\left(m^{\prime} n^{\prime}\right)} \\
V_{z}^{\left(l^{\prime}\right)}
\end{array}\right]
$$

where $Z$ denotes the mutual impedances between the testing and basis functions, and $V$ represents the excitation voltages due to the current source(s). Since the matrix entries corresponding to horizontal conductors, namely $Z_{x x}, Z_{x y}, Z_{y x}$, and $Z_{y y}$, have been written explicitly and treated already [16]-[18], for the sake of brevity, they are not given here. However, those entries associated with vertical metallization, $Z_{x z}, \quad Z_{z x}, \quad Z_{y z}, \quad Z_{z y}, \quad Z_{z z}$, are given here because the difficulties involved in, and the solutions proposed for, their evaluations require the explicit knowledge of these terms:

$$
\begin{aligned}
Z_{z x}^{\left(l^{\prime}, m n\right)}= & -j \omega\left\langle T_{z}^{\left(l^{\prime}\right)}, G_{z x}^{A} * B_{x}^{(m n)}\right\rangle \\
& +\frac{1}{j \omega}\left\langle T_{z}^{\left(l^{\prime}\right)}, \frac{\partial}{\partial z}\left[G_{x}^{q_{e}} * \frac{\partial B_{x}^{(m n)}}{\partial x}\right]\right\rangle \\
Z_{x z}^{\left(m^{\prime} n^{\prime}, l\right)}= & \frac{1}{j \omega}\left\langle T_{x}^{\left(m^{\prime} n^{\prime}\right)}, \frac{\partial}{\partial x}\left[G_{z}^{q_{e}} * \frac{\partial B_{z}^{(l)}}{\partial z}\right]\right\rangle \\
Z_{z y}^{\left(l^{\prime}, m n\right)}= & -j \omega\left\langle T_{z}^{\left(l^{\prime}\right)}, G_{z y}^{A} * B_{y}^{(m n)}\right\rangle \\
& +\frac{1}{j \omega}\left\langle T_{z}^{\left(l^{\prime}\right)}, \frac{\partial}{\partial z}\left[G_{y}^{q_{e}} * \frac{\partial B_{y}^{(m n)}}{\partial y}\right]\right\rangle \\
Z_{y z}^{\left(m^{\prime} n^{\prime}, l\right)=} & \frac{1}{j \omega}\left\langle T_{y}^{\left(m^{\prime} n^{\prime}\right)}, \frac{\partial}{\partial y}\left[G_{z}^{q_{e}} * \frac{\partial B_{z}^{(l)}}{\partial z}\right]\right\rangle \\
Z_{z z}^{\left(l^{\prime}, l\right)}= & -j \omega\left\langle T_{z}^{\left(l^{\prime}\right)}, G_{z z}^{A} * B_{z}^{(l)}\right\rangle \\
& +\frac{1}{j \omega}\left\langle T_{z}^{\left(l^{\prime}\right)}, \frac{\partial}{\partial z}\left[G_{z}^{q_{e}} * \frac{\partial B_{z}^{(l)}}{\partial z}\right]\right\rangle
\end{aligned}
$$

where $\langle$,$\rangle , and *$ denote inner product and convolution, respectively. The entries of the array $V$ have the same form except the basis function, which is a half-rooftop function with unit amplitude to model the current source.

\section{B. Closed-Form Green's Functions in the Spatial Domain}

Since the efficient evaluation of the MoM matrix entries $Z$ and $V$ in (8), requires the efficient calculation of the spatial-domain Green's functions $G^{A_{\mathrm{s}}} \mathrm{s}$ and $G^{q_{e}} \mathrm{~s}$, it would be instructive to give a brief description of the spatial-domain closed-form Green's functions. Moreover, the steps of the derivation of the closed-form Green's functions reveal the difficulties involved in the evaluation of the inner-product terms, (9)-(13).

It is well known that the Green's functions in planar layered media can be obtained analytically in closed forms only in the spectral domain. Therefore, the spatial-domain representation is usually based on the spectral-domain Green's functions through the Hankel transformation as

$$
G^{A, q_{e}}=\frac{1}{4 \pi} \int_{\mathrm{SI \Gamma}} d k_{\rho} k_{\rho} H_{0}^{(2)}\left(k_{\rho} \rho\right) \tilde{G}^{A, q_{e}}\left(k_{\rho}\right)
$$

where $G$ and $\tilde{G}$ are the Green's functions in the spatial- and spectral-domains, respectively, $H_{0}^{(2)}$ is the Hankel function of the second kind and SIP denotes the Sommerfeld integration path [22]. The Hankel transform of a spectral-domain Green's function cannot be evaluated analytically in general, except for a few trivial cases, and numerical implementation is computationally expensive because of the oscillatory nature of the kernel $H_{0}^{(2)}$ and the slow-converging nature of some of the spectral-domain Green's functions. Since the MoM matrix entries (8) require convolution integrals over the domains of the basis functions and require double-integrals over the domains of the testing functions, one needs to know the values of the spatial-domain Green's functions for hundreds of 
space coordinates, if not thousands. So, it would be inefficient to numerically implement the Hankel transformation for the spatial-domain application of the MoM [23]. Based on the fact that the Hankel transform of $e^{-j k_{z}|z|} / k_{z}$ is known analytically, called the Sommerfeld identity, and given as

$$
\frac{e^{-j k r}}{r}=\frac{1}{2 j} \int_{\mathrm{SIP}} d k_{\rho} k_{\rho} H_{0}^{(2)}\left(k_{\rho} \rho\right) \frac{e^{-j k_{z}|z|}}{k_{z}} .
$$

Chow et al., recognized that if the spectral-domain Green's functions are approximated in terms of exponentials, the Hankel transformation can be evaluated analytically [8]. This approach puts the emphasis on the algorithms for exponential approximation, among those can be named the Prony, the pencil of functions methods, and their variants [9]-[11]. The details of the exponential approximation technique used throughout this paper, namely the GPOF, are not given here because it is both out of the scope of this paper and welldocumented in literature [10]. However, a representative form of the spectral-domain Green's functions is included to help explain the procedure for approximating them in terms of exponentials

$$
\tilde{G} \propto \frac{1}{j 2 k_{z_{i}}}\left[A\left(k_{\rho}, z^{\prime}\right) e^{-j k_{z_{i}} z}+B\left(k_{\rho}, z^{\prime}\right) e^{j k_{z_{i}} z}\right]
$$

where subscript $i$ denotes the source region, and $A$ and $B$ are amplitudes of positive- and negative-going waves, whose explicit expressions can be found in [7]. It should be noted that the coefficients $A$ and $B$ are the exponential functions of $z^{\prime}$.

The exponential approximation begins with sampling the function to be approximated, and then the algorithm for exponential approximation is employed for the sampled values of the function. In other words, one needs to know the values of the function at the points of samples, which requires fixing the parameters, like $z$ and $z^{\prime}$ in (16). After having sampled the spectral-domain Green's function to be approximated, apart from the term $1 / j 2 k_{z_{i}}$, the GPOF method is used to obtain the exponential approximation of the function, which results in an approximation as follows:

$$
\tilde{G}^{A, q_{e}} \cong \frac{1}{j 2 k_{z_{i}}} \sum_{m=1}^{N} a_{m} e^{-b_{m} k_{z_{i}}}
$$

Hence, this approximating function can be transformed to the spatial domain analytically via the Sommerfeld identity (15), resulting in the following spatial-domain expression, also called the closed-form Green's functions:

$$
G^{A, q_{e}} \cong \sum_{m=1}^{N} a_{m} \frac{e^{-j k_{i} r_{m}}}{r_{m}}
$$

where $r_{m}=\sqrt{\rho^{2}-b_{m}^{2}}$. It should be noted that the sampling of the spectral-domain Green's functions should be performed along the SIP or along a path legitimately deformed from the SIP, details of which can be found in [8], [13]. In this paper, a deformed path from the SIP as depicted in Fig. 3 has been employed, consisting of three connected paths denoted as $C_{a p 1}, C_{a p 2}$, and $C_{a p 3}$, respectively, and described by the

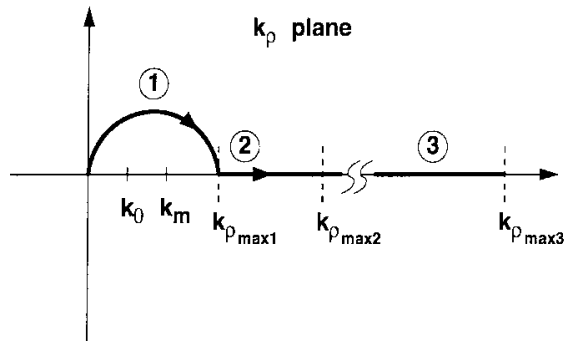

Fig. 3. Three-level GPOF sampling paths.

following parametric equations:

For $\quad C_{a p 3}, k_{z_{i}}=-j k_{i}\left[T_{\circ 1}+T_{\circ 2}+t\right], \quad 0 \leq t \leq T_{\circ 3}$

For $\quad C_{a p 2}, k_{z_{i}}=-j k_{i}\left[T_{\circ 1}+t\right], \quad 0 \leq t \leq T_{\circ 2}$

For $C_{a p 1}, k_{z_{i}}=k_{i}\left[-j t+\left(1-\frac{t}{T_{\circ 1}}\right)\right], \quad 0 \leq t \leq T_{\circ 1}$

where $t$ is the running variable sampled uniformly on the corresponding ranges, $T_{\mathrm{O} 1}, T_{\mathrm{O} 2}$, and $T_{\mathrm{O} 3}$. This approach is named, hereafter, as the three-level approach, which is an extension of the two-level approach introduced recently [13], so its details are not included in this paper. Because the spectral-domain Green's functions might have fast variations locally, and because the GPOF method requires uniform sampling along the range of approximation, the use of a multilevel approach prevents taking thousands of samples. However, it is not necessary to use the three-level approach for smooth functions, for which one may use the two-level or one-level approach, simply by setting $T_{\mathrm{\circ} 3}$ to zero or $T_{\circ 2}$ and $T_{\mathrm{o} 3}$ to zero, respectively.

\section{DifFiculties AND SOlutions}

The use of the closed-form Green's functions in conjunction with the MoM has been demonstrated to improve the computational efficiency of the MoM when applied to simple geometries like those consisting of only horizontal conductors, or geometries with simplifying assumptions. After having improved the computational efficiency and robustness of the derivation of the closed-form Green's functions, the natural step toward the goal of developing an accurate and efficient em simulator is to study this approach for general geometries. A preliminary study shows that the application of the MoM in conjunction with the closed-form Green's functions is not as straightforward as its applications to only horizontal geometries, i.e., there are some difficulties in cases of vertical metallizations.

These difficulties originates from the way the closed-form Green's functions are derived, more specific, from the exponential approximation of the spectral-domain Green's functions. In Section II, the representative form of the spectraldomain Green's functions is given and it is stated that the parameters, $z$ and $z^{\prime}$, have to be fixed in order to be able to sample the function over the range of approximation. In other words, the exponential approximation is valid for only 
those fixed values of the parameters and so is the closedform Green's functions. For horizontal conductors, fixing $z$ and $z^{\prime}$ does not pose a problem because the conductors are situated on constant $z$-planes requiring the Green's functions to be evaluated at these planes only. Therefore, one can fix these parameters prior to the derivation of the closedform Green's functions, and use these Green's functions for those values of the parameters only. However, the evaluations of the MoM matrix entries corresponding to the vertical metallizations require convolution integrals and inner-product integrals (9)-(13), which are to be integrated over $z$ and/or $z^{\prime}$. So, the closed-form Green's functions, derived as described in the previous section, cannot be used efficiently in the evaluation of such matrix entries.

This difficulty can be eliminated by recognizing that the amplitudes of the up- and down-going waves in the spectraldomain Green's functions are the exponential functions of $z^{\prime}$ that can be factored out (see the Appendix). As an example, the spectral-domain Green's function for the scalar potential due to a vertical electric dipole can be written as

$$
\begin{aligned}
\tilde{G}_{z}^{q}= & \frac{1}{2 j k_{z_{i}} \epsilon_{i}}\left\{e^{-j k_{z_{i}}\left|z-z^{\prime}\right|}+\tilde{R}_{\mathrm{TM}}^{i, i-1} M_{i}^{\mathrm{TM}}\right. \\
& \cdot\left[-e^{-j k_{z_{i}}\left(z+z^{\prime}\right)}+\tilde{R}_{\mathrm{TM}}^{i, i+1} e^{-j k_{z_{i}}\left(z-z^{\prime}+2 d_{i}\right)}\right] \\
& +\tilde{R}_{\mathrm{TM}}^{i, i+1} M_{i}^{\mathrm{TM}} \\
& \left.\cdot\left[-e^{-j k_{z_{i}}\left(2 d_{i}-z-z^{\prime}\right)}+\tilde{R}_{\mathrm{TM}}^{i, i-1} e^{-j k_{z_{i}}\left(z^{\prime}-z+2 d_{i}\right)}\right]\right\}
\end{aligned}
$$

after having substituted the amplitudes of the up- and downgoing waves. Note that $\tilde{R}_{\mathrm{TM}}$ s and $M_{i}^{\mathrm{TM}}$ are not functions of $z$ and $z^{\prime}$, and their explicit expressions can be found in [6], [7]. A brief study of (22) shows that there are two approaches to overcome the difficulty, which are 1) application of the exponential approximation to each amplitude and 2) integration over $z$ and $z^{\prime}$ performed analytically in the spectral domain, after which the exponential approximation is employed. In the first approach, one needs to deal with each term in (22) separately; the first one is the direct term with unity amplitude, so there is no need for approximation, and the rest have amplitudes as functions of $k_{\rho}$. In other words, approximation of $\tilde{G}_{z}^{q}$ in terms of complex exponentials with the exponents including $z$ and $z^{\prime}$ explicitly requires to approximate only the amplitude functions of the four exponentials in (22), namely $\tilde{R}_{\mathrm{TM}}^{i, i-1} M_{i}^{\mathrm{TM}}, \tilde{R}_{\mathrm{TM}}^{i, i-1} M_{i}^{\mathrm{TM}} \tilde{R}_{\mathrm{TM}}^{i, i+1}$, and $\tilde{R}_{\mathrm{TM}}^{i, i+1} M_{i}^{\mathrm{TM}}$. The cost of having $z$ and $z^{\prime}$ explicitly in the approximation of a Green's function is to apply the GPOF method three times more and use exponentials four times more as compared to approximating the same Green's function as a whole. The second solution is based on the fact that $z$ and $z^{\prime}$ dependence of the spectral-domain Green's functions is always in exponential form and analytically integrable over $z$ and $z^{\prime}$ for most basis functions. Therefore, the integration over $z$ and $z^{\prime}$, which are due to testing and convolution integrals along a vertical metallization, respectively, can be evaluated analytically if the spatial-domain Green's functions in the inner-product expressions are written as the inverse transforms of their spectral-domain representations. Then, the exponential approximation procedure is implemented on the resulting spectral-domain function. This approach eliminates the application of the exponential approximation for each term in the spectral-domain Green's function. However, it requires the application of exponential approximation as many times as the number of testing functions, or number of basis functions, or number of basis times testing functions on the vertical metallization for the inner-product terms involving $z$ or $z^{\prime}$ or $z$ and $z^{\prime}$ integrations, respectively. Although the second approach seems to employ the exponential approximation algorithm more than the first one, it is more efficient for short vertical metallizations for which only a few basis functions are used. For one basis function on the vertical metallization which is usually sufficient for a practical geometry, the number of exponential approximation in the second approach is less than that in the first approach, and moreover, it requires less number of exponentials even for several basis functions. Also note that some of the commercial $\mathrm{em}$-simulation softwares, like em from SONNET, use only one basis function along a vertical metallization.

The first approach described above is quite straightforward, where one needs to write the spectral-domain Green's functions in terms of exponentials of $z$ and $z^{\prime}$ with complex coefficients and to apply the GPOF method for each complex coefficient. Therefore, there is no need to give further details for this approach. On the other hand, since the application of the second approach requires some work in the spectraldomain, it would be instructive to give the procedure and the following details:

1) write the spectral-domain Green's functions into the sum of exponentials of $z$ and $z^{\prime}$ with complex coefficients;

2) write the spatial-domain Green's functions in the MoM matrix entries as the inverse transform of the spectraldomain counterpart using (14);

3) evaluate the integrals over $z$ and/or $z^{\prime}$ variables analytically;

4) approximate the resultant expression by complex exponentials via the GPOF method;

5) transform the whole expression into the spatial domain via the Sommerfeld identity (15), getting an auxiliary function $F_{x, z z, z x}^{q, A}$ which has the same form as (18);

6) evaluate the remaining inner-product integrals analytically in the spatial domain.

Exponential approximations of the integrated spectraldomain Green's functions with the GPOF method (item 4) should be performed with care because it has been observed that the functions obtained after evaluating $z$ and $z^{\prime}$ integrals may contain peaks for intermediate values of $k_{\rho}$. Therefore, to capture such behaviors efficiently, the two-level approximation scheme [13] is extended to three levels for these terms. Hence, it is guaranteed that the spectral-domain auxiliary functions are approximated successfully. It should also be noted that addition of multiple vertical strips will not increase the computational cost of this technique, provided that all vertical strips employ the same number of basis functions. This is because the MoM matrix entries corresponding to the basis functions on a vertical strip are obtained as a function of $\rho$ and because the domains of integrations along the vertical strips are the same. In other words, once the interaction 
between a basis and a testing function on a vertical strip is calculated, the same expression can be used with a different value of $\rho$ for the calculation of the reaction of the same basis function and a testing function (or vice versa) located on an another vertical strip at a distance of $\rho$.

To demonstrate this technique on an example, the innerproduct term containing an integration on $z$-variable is evaluated as shown in (23) at the bottom of this page.

The first step of the procedure is to write the spectral-domain Green's function $\tilde{G}_{z x}^{A}$ (38), in the form of (44), where $z$ and $z^{\prime}$ dependences are explicit. Then, the spatial-domain Green's function $G_{z x}^{A}$ in (23) is replaced by the inverse transform of the spectral-domain Green's function $\tilde{G}_{z x}^{A}$, (44). Hence, the inner-product term in (23) becomes

$$
\begin{aligned}
\left\langle T_{z}^{\left(l^{\prime}\right)}, G_{z x}^{A} * B_{x}^{(m n)}\right\rangle & \\
= & \iint d z d y T_{z}^{\left(l^{\prime}\right)}(y) T_{z}^{\left(l^{\prime}\right)}(z) \iint d x^{\prime} d y^{\prime} \\
& \cdot\left\{\frac{1}{4 \pi} \int_{\mathrm{SIT}} d k_{\rho} k_{\rho} H_{0}^{(2)}\left(k_{\rho}\left|\rho-\rho^{\prime}\right|\right) \tilde{G}_{z x}^{A}\left(k_{\rho}, z\right)\right\} \\
& \cdot B_{x}^{(m n)}\left(x^{\prime}, y^{\prime}\right)
\end{aligned}
$$

where the separability of the basis functions is utilized, $T_{z}^{\left(l^{\prime}\right)}(y, z)=T_{z}^{\left(l^{\prime}\right)}(y) T_{z}^{\left(l^{\prime}\right)}(z)$. After changing the order of integrations, the following auxiliary function is defined and cast into closed form via the Sommerfeld identity:

$$
\begin{aligned}
F_{z x}^{A} \stackrel{\text { def }}{=} & \int d z T_{z}^{\left(l^{\prime}\right)}(z) \frac{1}{4 \pi} \int_{\mathrm{SIP}} \\
& d k_{\rho} k_{\rho} H_{0}^{(2)}\left(k_{\rho}\left|\rho-\rho^{\prime}\right|\right) \tilde{G}_{z x}^{A}\left(k_{\rho}, z\right) \\
\cong & \frac{1}{4 \pi} \int_{\operatorname{SIP}} d k_{\rho} k_{\rho} H_{0}^{(2)}\left(k_{\rho}\left|\rho-\rho^{\prime}\right|\right) \\
& \cdot \operatorname{GPOF}\left\{\int d z T_{z}^{\left(l^{\prime}\right)}(z) \tilde{G}_{z x}^{A}\left(k_{\rho}, z\right)\right\}
\end{aligned}
$$

where GPOF \{\} designates the approximation process with complex exponentials via the GPOF method. Note that $\tilde{G}_{z x}^{A}$ has a multiplicative term of $-j k_{x}$, which is equivalent to $\partial / \partial x$ in the spatial domain. Therefore, the integral of the Green's function under the GPOF \{\} operator is approximated with complex exponentials without $-j k_{x}$ term, resulting in the following inner product expression:

$$
\begin{gathered}
\left\langle T_{z}^{\left(l^{\prime}\right)}, G_{z x}^{A} * B_{x}^{(m n)}\right\rangle \\
=-\int d y T_{z}^{\left(l^{\prime}\right)}(y) \iint d x^{\prime} d y^{\prime} \\
\cdot B_{x}^{(m n)}\left(x^{\prime}, y^{\prime}\right) \frac{\partial}{\partial x^{\prime}} F_{z x}^{A} .
\end{gathered}
$$

The derivative of $F_{z x}^{A}$ with respect to $x^{\prime}$ is carried over the basis function by using the chain rule

$$
\begin{aligned}
\frac{\partial}{\partial x^{\prime}}\left[B_{x}^{(m n)}\left(x^{\prime}, y^{\prime}\right) F_{z x}^{A}\right]= & \frac{\partial}{\partial x^{\prime}} B_{x}^{(m n)}\left(x^{\prime}, y^{\prime}\right) F_{z x}^{A} \\
& +B_{x}^{(m n)}\left(x^{\prime}, y^{\prime}\right) \frac{\partial}{\partial x^{\prime}} F_{z x}^{A}
\end{aligned}
$$

and the inner-product (23) reduces to

$$
\begin{aligned}
\left\langle T_{z}^{\left(l^{\prime}\right)}, G_{z x}^{A} * B_{x}^{(m n)}\right\rangle & \\
= & -\left.\int d y T_{z}^{\left(l^{\prime}\right)}(y) \int d y^{\prime}\left[B_{x}^{(m n)}\left(x^{\prime}, y^{\prime}\right) F_{z x}^{A}\right]\right|_{\Omega_{B}} \\
& +\int d y T_{z}^{\left(l^{\prime}\right)}(y) \iint d x^{\prime} d y^{\prime} F_{z x}^{A} \frac{\partial}{\partial x^{\prime}} B_{x}^{(m n)}\left(x^{\prime}, y^{\prime}\right) .
\end{aligned}
$$

Following the substitutions $x-x^{\prime}=u$ and $y-y^{\prime}=v$, the inner-product expression (28) is further simplified to

$$
\begin{aligned}
& \left\langle T_{z}^{\left(l^{\prime}\right)}, G_{z x}^{A} * B_{x}^{(m n)}\right\rangle \\
& =\left.\int d v F_{z x}^{A} \int d y\left[T_{z}^{\left(l^{\prime}\right)}(y) B_{x}^{(m n)}(x-u, y-v)\right]\right|_{\Omega_{B}} \\
& \quad+\iint d u d v F_{z x}^{A} \int d y T_{z}^{\left(l^{\prime}\right)}(y) \frac{\partial}{\partial x^{\prime}} B_{x}^{(m n)}(x-u, y-v)
\end{aligned}
$$

where $x=x_{s p}, x$-coordinate of the vertical metallization and the integrals over $y$ are evaluated analytically. Note that the first term in (29) drops for the rooftop basis functions, but it must be evaluated for the basis functions that are not zero at the boundaries such as half-rooftop functions. Evaluations of the other inner-products given in (9)-(13) are performed similarly, so they are not given here for the sake of brevity.

\section{RESULTS AND DisCUSSIONS}

In this section, application of the formulation presented in this paper is demonstrated on some practical geometries and the results are compared with those obtained from the commercial EM analysis program em, version 3.0 (SONNET Software, Inc.). The main difference between $\mathrm{em}$ and this formulation is that em puts the circuit in a conducting box whose default wall conductivities are infinite [24]. Analyzing radiating structures in em requires adding loss to the system, but with the formulation presented here these structures can be characterized better, in the sense of accuracy and computational efficiency. One can refer to [25] for general information on em software. In all of the geometries showing the test circuits, the arrows on the port transmission lines show the reference planes used in the calculation and in the de-embedding of the circuit parameters.

$$
\left\langle T_{z}^{\left(l^{\prime}\right)}, G_{z x}^{A} * B_{x}^{(m n)}\right\rangle=\iint d z d y T_{z}^{\left(l^{\prime}\right)}(y, z) \iint d x^{\prime} d y^{\prime} G_{z x}^{A}\left(x-x^{\prime}, y-y^{\prime}, z, z^{\prime}=\text { cons. }\right) B_{x}^{(m n)}\left(x^{\prime}, y^{\prime}\right) .
$$




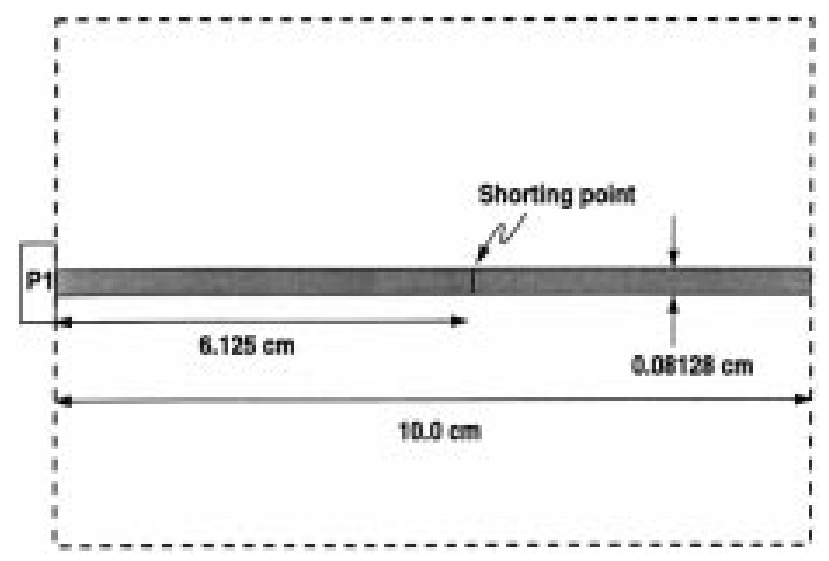

Fig. 4. Geometry of the microstrip line with shorting strip $\left(\varepsilon_{r}=4.0, h=\right.$ see the text).

As a first example, a simple microstrip line short-circuited with a shorting p-i-n, as shown in Fig. 4, is considered, where the shorting pin is modeled as a narrow rectangular strip placed between the microstrip line and the ground plane. Note that the same model is also used in the em simulation for the purpose of comparison. However, it should be stated that a more realistic shorting pin can be modeled by placing the vertical strips in a form of rectangular cylinder both in $\mathrm{em}$ and in this approach. The dielectric constant of the substrate is chosen to be 4.0, and two different values of the substrate thickness are used in the analysis, 8 and 80 mil. The microstrip line is excited with a current source from the left end, and is left open-circuited at the right end. To simulate the unity current-source excitation in $\mathrm{em}$, the values of the voltage source and the input impedance in em are selected equal and very large with respect to the characteristic impedance of the line, which is the Norton equivalent of the current source with unity amplitude. The current distributions on the microstrip line are obtained and shown in Figs. 5 and 6, with the results obtained from the em software, denoted by $\mathrm{em}$ in these figures. As can be seen from the figures, the results agree very well for a thin substrate case but, as the substrate thickness increases, the result of $\mathrm{em}$ starts to deviate because em version 3.0 assumes constant current distributions on vertical connections [24]. Note that three shorting pins have been employed for the thick substrate case to demonstrate the use of the formulation for multiple vertical metallizations (see Fig. 6).

The next example is a radiating structure-a microstrip patch antenna with a shorting post, printed on a substrate with the thickness of $0.152 \mathrm{~cm}$ and with the relative permittivity of 2.43, as shown in Fig. 7. Placing shorting posts in a microstrip patch antenna shifts the resonant frequency of the antenna without effecting the radiation pattern significantly [26]-[28]. Therefore, it can be utilized in the design of a dual-frequency microstrip antenna. Moreover, if these shorting posts are made by using $\mathrm{p}-\mathrm{i}-\mathrm{n}$ or varactor diodes, then a frequency-agile antenna is obtained by electrically activating the diodes. In this example, the amount of frequency shift in the resonant frequency of a patch antenna is assessed, and demonstrated for several positions of the shorting post in Fig. 8, where

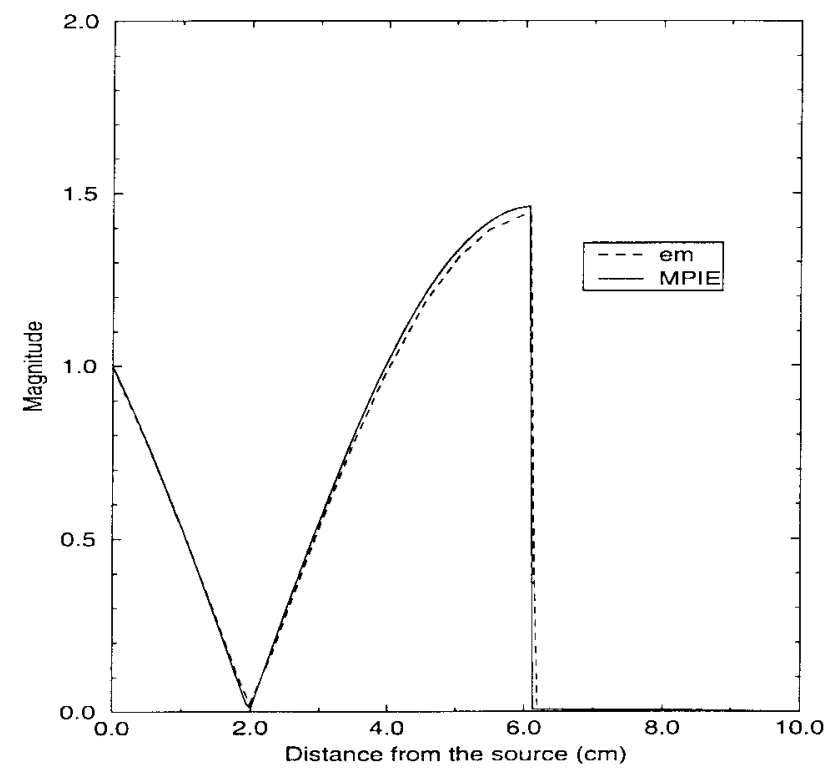

Fig. 5. Current distribution on the shorted microstrip line $(h=8$ mil, one shorting pin).

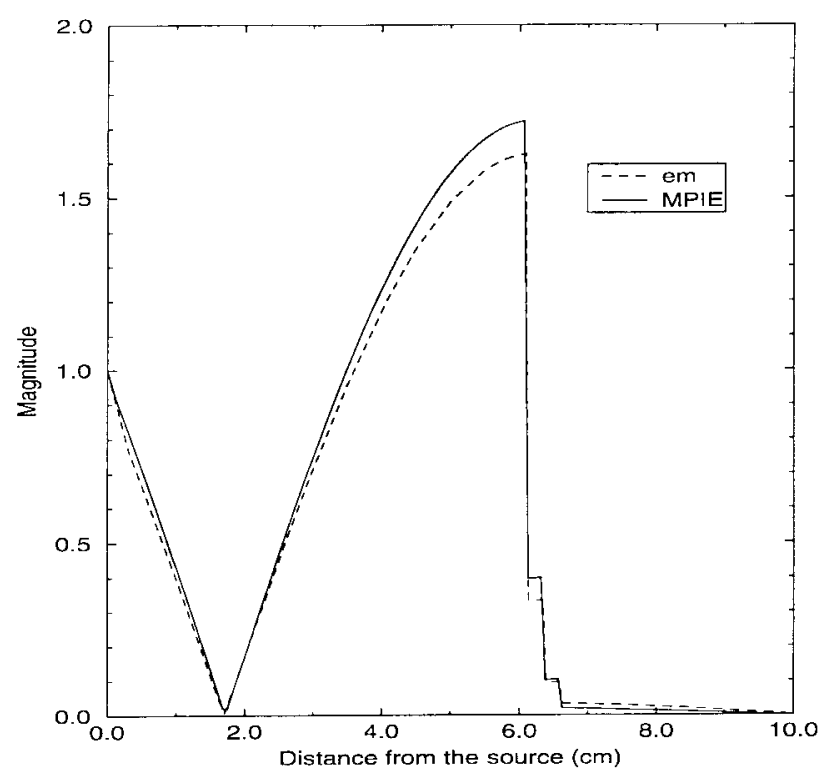

Fig. 6. Current distribution on the shorted microstrip line $(h=80 \mathrm{mil}$, three shorting pins).

the normalized position of the shorting post is defined as $x_{n}=(x-3.81) / 3.81$. The results obtained by the method presented in this paper are compared to those measured and to those obtained by the cavity model, and the agreement is quite good considering the different types of feeding structures, microstrip-line feed for this case, and probe feed for the experimental and cavity model [27]. Note that the unloaded resonant frequency of the antenna is approximately $1237 \mathrm{MHz}$, and it also corresponds to the position of the shorting post at the middle of the patch. This is because the dominant modes in this patch antenna, $\mathrm{TM}_{01}$ and $\mathrm{TM}_{10}$, have almost zero electric field in $z$-direction at the middle of the patch, hence, the shorting post at this position will have negligible effect on the resonant frequency. 


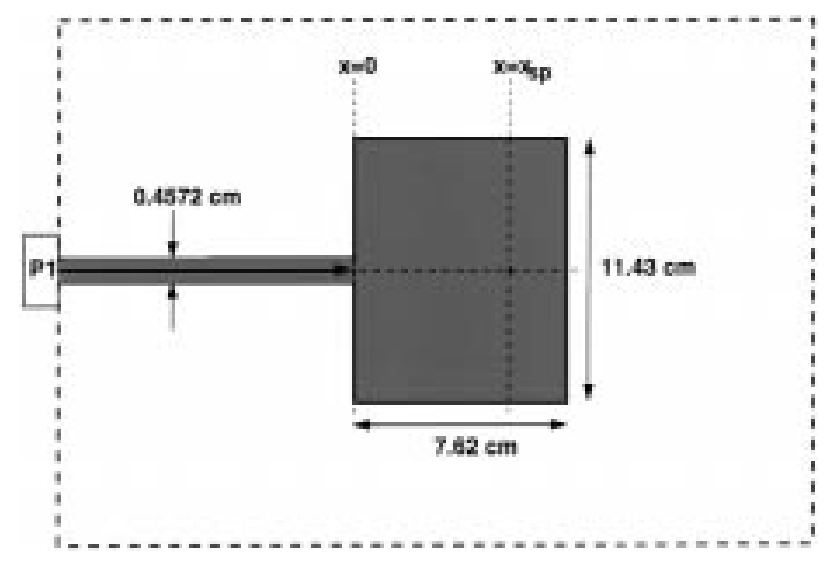

Fig. 7. Geometry of the microstrip patch antenna with a shorting pin $\left(\varepsilon_{r}=2.43, h=0.152 \mathrm{~cm}\right)$.

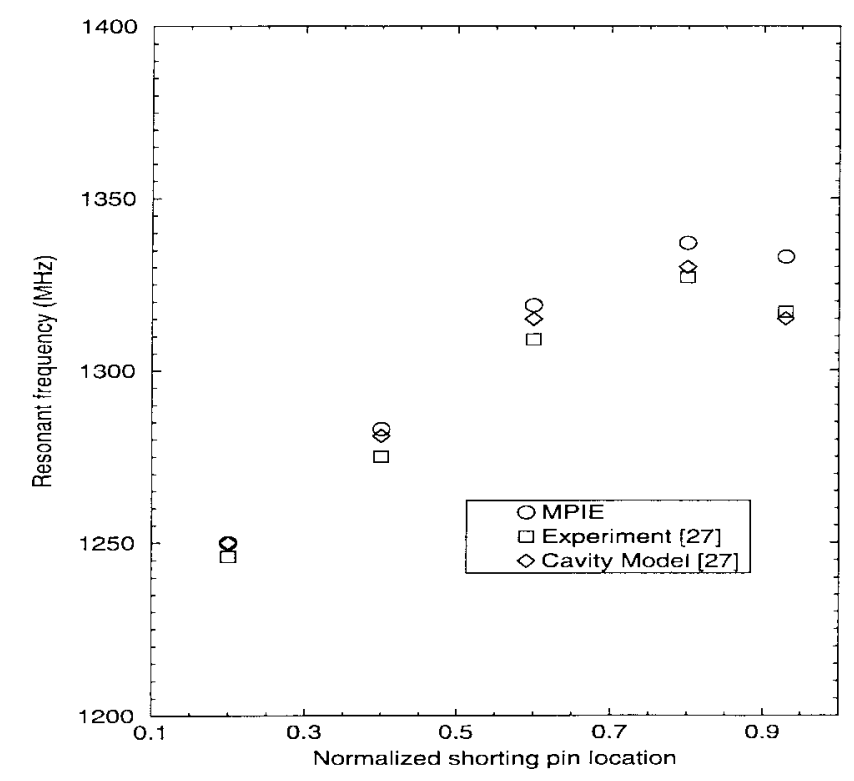

Fig. 8. Resonant frequency versus normalized shorting-pin location for the patch antenna.

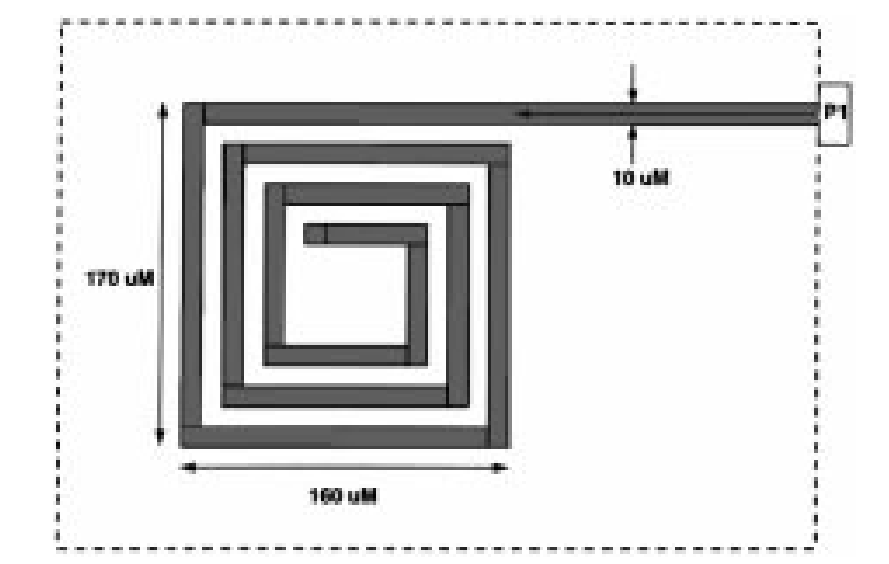

Fig. 9. Geometry of the square-spiral MMIC inductor $\left(\varepsilon_{r}=12.9, h=100 \mu \mathrm{m}\right)$.

Finally, a square-spiral MMIC inductor terminated in a short circuit shown in Fig. 9, printed on a substrate with a thickness of $100 \mu \mathrm{m}$ and with the dielectric constant of

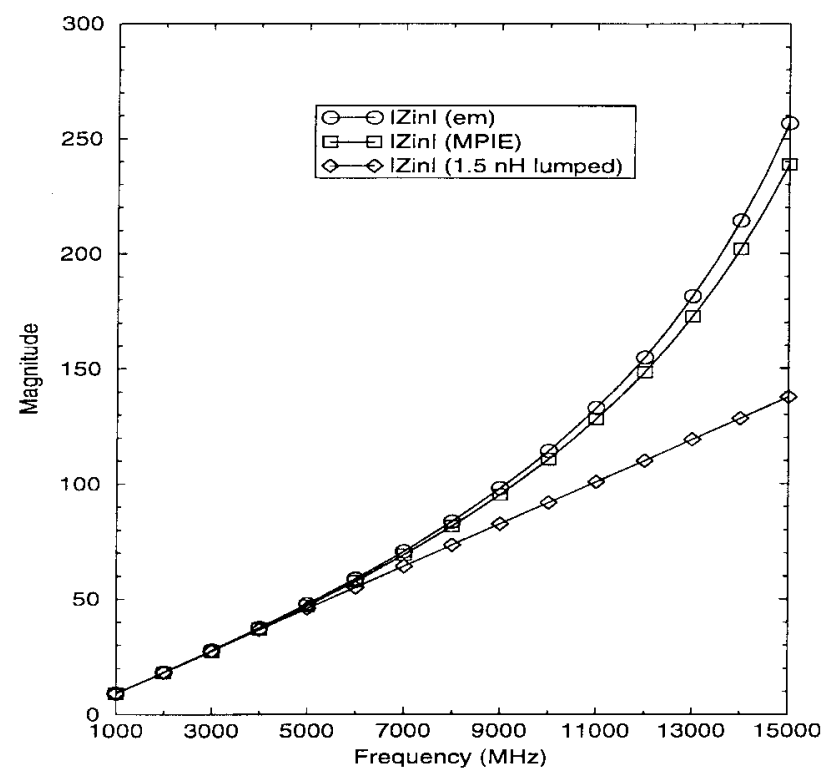

Fig. 10. Magnitude of $Z_{\text {in }}$ of the MMIC inductor.

12.9, is analyzed. The magnitude and the phase of the input impedance, seen from the reference plane, is calculated and the magnitude is plotted in Fig. 10 as a function of frequency and the phase is equal to $90^{\circ}$ through the range of frequency. It is observed that the magnitude of the input impedance deviates from the ideal behavior of an inductor, as the frequency is increased, because of the self-resonance of the structure.

It should be noted that the model employed for vertical metallizations, namely the planar strip, is not the most accurate one. One can instead use circular cylindrical or square cylindrical via models within this formulation. But, since the main goal of this paper is to demonstrate the use of the closed-form Green's functions in conjunction with the MoM for geometries with vertical metallizations, the simplest model is employed to convey the ideas clearly. In addition, although the examples provided here only employ shorting pins to the ground plane, it is equally easy to implement vias between any two consecutive layers, like in the case of an inductor with an air-bridge.

\section{CONCLUSION}

Efficient derivation of the closed-form Green's functions in the spatial-domain has improved the computational efficiency of the MoM for planar geometries in multilayer media. However, there have been some difficulties in the use of these closed-form Green's functions in the analysis of geometries with vertical metallizations. In this paper, the authors have discussed these difficulties and have proposed two techniques to overcome these problems. It is observed that one of these technique, the first one, is more efficient for long vertical metallizations requiring several basis functions, and the other approach is more efficient for short vertical metallizations. Since the implementation of the first one is rather simple and straightforward, the authors have given the formulation of the second approach in detail. The for- 
mulation is applied to some realistic geometries, for which the scattering parameters and the relevant circuit parameters are extracted and compared to those obtained from a well-known commercial software, em, from SONNET. The formulation presented here is not limited to thin substrates, in other words, to short vertical metallizations as in the case of em version 3.0, and is capable of incorporating additional vertical metallizations with almost no computational cost. Therefore, this approach is very suitable for the purpose of optimization of a geometry involving vertical metallizations.

\section{APPENDIX}

\section{SPECTRAL-DOMAIN GREEN'S FUnCTIONS}

As discussed in Section II, some of the spectral-domain Green's functions need to be cast into a specific form, where $z$ and $z^{\prime}$ dependences are factored out. The only Green's function that does not require this factorization is $\tilde{G}_{x x}^{A}$, because it is always evaluated at constant $z$ - and $z^{\prime}$-planes. Therefore, the components of the spectral-domain Green's functions, except $\tilde{G}_{x x}^{A}$, are cast into the suitable form for the formulation presented in the paper, and are given here for convenience. For a detailed derivation of the spectral-domain Green's functions, one can refer to [7]. Since the origin is located at the bottom of the source layer (denoted by the subscript $i$ ) for the application of the MoM, the coordinates used in the derivation of the Green's functions in [7] is transformed from $z$ to $z-z^{\prime}$, and $h$ to $z^{\prime}$.

\section{A. The Green's Function $\tilde{G}_{z}^{q}$}

$$
\begin{aligned}
\tilde{G}_{z}^{q}= & \frac{1}{2 j k_{z_{i}} \epsilon_{i}} \\
& \cdot\left[e^{-j k_{z_{i}}\left|z-z^{\prime}\right|}+C_{v}^{e} e^{-j k_{z_{i}}\left(z-z^{\prime}\right)}+D_{v}^{e} e^{j k_{z_{i}}\left(z-z^{\prime}\right)}\right]
\end{aligned}
$$

where

$$
\begin{aligned}
& C_{v}^{e}=\tilde{R}_{\mathrm{TM}}^{i, i-1} M_{i}^{\mathrm{TM}}\left[-e^{-2 j k_{z_{i}} z^{\prime}}+\tilde{R}_{\mathrm{TM}}^{i, i+1} e^{-2 j k_{z_{i}} d_{i}}\right] \\
& D_{v}^{e}=\tilde{R}_{\mathrm{TM}}^{i, i+1} M_{i}^{\mathrm{TM}}\left[-e^{-2 j k_{z_{i}}\left(d_{i}-z^{\prime}\right)}+\tilde{R}_{\mathrm{TM}}^{i, i-1} e^{-2 j k_{z_{i}} d_{i}}\right]
\end{aligned}
$$

Substituting (31) and (32) into (30), the following expression is obtained;

$$
\begin{aligned}
\tilde{G}_{z}^{q}= & \frac{1}{2 j k_{z_{i}} \epsilon_{i}}\left\{e^{-j k_{z_{i}}\left|z-z^{\prime}\right|}+\tilde{R}_{\mathrm{TM}}^{i, i-1} M_{i}^{\mathrm{TM}}\right. \\
& \cdot\left[-e^{-j k_{z_{i}}\left(z+z^{\prime}\right)}+\tilde{R}_{\mathrm{TM}}^{i, i+1} e^{-j k_{z_{i}}\left(z-z^{\prime}+2 d_{i}\right)}\right] \\
& +\tilde{R}_{\mathrm{TM}}^{i, i+1} M_{i}^{\mathrm{TM}} \\
& \left.\cdot\left[-e^{-j k_{z_{i}}\left(2 d_{i}-z-z^{\prime}\right)}+\tilde{R}_{\mathrm{TM}}^{i, i-1} e^{-j k_{z_{i}}\left(z^{\prime}-z+2 d_{i}\right)}\right]\right\}
\end{aligned}
$$

\section{B. The Green's Function $\tilde{G}_{z z}^{A}$}

$$
\begin{aligned}
\widetilde{G}_{z z}^{A}= & \frac{\mu_{i}}{2 j k_{z_{i}}} \\
& \cdot\left[e^{-j k_{z_{i}}\left|z-z^{\prime}\right|}+A_{v}^{e} e^{-j k_{z_{i}}\left(z-z^{\prime}\right)}+B_{v}^{e} e^{j k_{z_{i}}\left(z-z^{\prime}\right)}\right]
\end{aligned}
$$

where

$$
\begin{aligned}
& A_{v}^{e}=\tilde{R}_{\mathrm{TM}}^{i, i-1} M_{i}^{\mathrm{TM}}\left[e^{-2 j k_{z_{i}} z^{\prime}}+\tilde{R}_{\mathrm{TM}}^{i, i+1} e^{-2 j k_{z_{i}} d_{i}}\right] \\
& B_{v}^{e}=\tilde{R}_{\mathrm{TM}}^{i, i+1} M_{i}^{\mathrm{TM}}\left[e^{-2 j k_{z_{i}}\left(d_{i}-z^{\prime}\right)}+\tilde{R}_{\mathrm{TM}}^{i, i-1} e^{-2 j k_{z_{i}} d_{i}}\right]
\end{aligned}
$$

Substituting (35) and (36) into (34), the following expression is obtained:

$$
\begin{aligned}
\tilde{G}_{z z}^{A}= & \frac{\mu_{i}}{2 j k_{z_{i}}}\left\{e^{-j k_{z_{i}}\left|z-z^{\prime}\right|}+\tilde{R}_{\mathrm{TM}}^{i, i-1} M_{i}^{\mathrm{TM}}\right. \\
& \cdot\left[e^{-j k_{z_{i}}\left(z+z^{\prime}\right)}+\tilde{R}_{\mathrm{TM}}^{i, i+1} e^{-j k_{z_{i}}\left(z-z^{\prime}+2 d_{i}\right)}\right] \\
& +\tilde{R}_{\mathrm{TM}}^{i, i+1} M_{i}^{\mathrm{TM}} \\
& \left.\cdot\left[e^{-j k_{z_{i}}\left(2 d_{i}-z-z^{\prime}\right)}+\tilde{R}_{\mathrm{TM}}^{i, i-1} e^{-j k_{z_{i}}\left(z^{\prime}-z+2 d_{i}\right)}\right]\right\} .
\end{aligned}
$$

C. The Green's Function $\tilde{G}_{z x}^{A}$

$$
\begin{aligned}
\tilde{G}_{z x}^{A}= & -\frac{\mu_{i}}{2 j k_{z_{i}}}\left[\frac{k_{x} k_{z_{i}}}{k_{\rho}^{2}}\left(A_{h}^{e}+B_{h}^{e}\right) e^{j k_{z_{i}}\left(z-z^{\prime}\right)}\right. \\
& \left.+\frac{k_{x} k_{z_{i}}}{k_{\rho}^{2}}\left(D_{h}^{e}-C_{h}^{e}\right) e^{-j k_{z_{i}}\left(z-z^{\prime}\right)}\right]
\end{aligned}
$$

where

$$
\begin{aligned}
A_{h}^{e} & =\tilde{R}_{\mathrm{TE}}^{i, i+1} M_{i}^{\mathrm{TE}}\left[e^{-2 j k_{z_{i}}\left(d_{i}-z^{\prime}\right)}+\tilde{R}_{\mathrm{TE}}^{i, i-1} e^{-2 j k_{z_{i}} d_{i}}\right] \\
B_{h}^{e} & =\tilde{R}_{\mathrm{TM}}^{i, i+1} M_{i}^{\mathrm{TM}}\left[e^{-2 j k_{z_{i}}\left(d_{i}-z^{\prime}\right)}-\tilde{R}_{\mathrm{TM}}^{i, i-1} e^{-2 j k_{z_{i}} d_{i}}\right] \\
C_{h}^{e} & =\tilde{R}_{\mathrm{TE}}^{i, i-1} M_{i}^{\mathrm{TE}}\left[e^{-2 j k_{z_{i}} z^{\prime}}+\tilde{R}_{\mathrm{TE}}^{i, i+1} e^{-2 j k_{z_{i}} d_{i}}\right] \\
D_{h}^{e} & =\tilde{R}_{\mathrm{TM}}^{i, i-1} M_{i}^{\mathrm{TM}}\left[-e^{-2 j k_{z_{i}} z^{\prime}}+\tilde{R}_{\mathrm{TM}}^{i, i+1} e^{-2 j k_{z_{i}} d_{i}}\right] .
\end{aligned}
$$

Substituting (39)-(42) into (38), the following expression is obtained:

$$
\begin{aligned}
\tilde{G}_{z x}^{A}= & -\frac{\mu_{i}}{2 j k_{z_{i}}}\left\{\frac { k _ { x } k _ { z _ { i } } } { k _ { \rho } ^ { 2 } } \left(\tilde{R}_{\mathrm{TE}}^{i, i+1} M_{i}^{\mathrm{TE}}\right.\right. \\
& \cdot\left[e^{-j k_{z_{i}}\left(2 d_{i}-z^{\prime}-z\right)}+\tilde{R}_{\mathrm{TE}}^{i, i-1} e^{-j k_{z_{i}}\left(2 d_{i}+z^{\prime}-z\right)}\right] \\
& +\tilde{R}_{\mathrm{TM}}^{i, i+1} M_{i}^{\mathrm{TM}} \\
& \left.\cdot\left[e^{-j k_{z_{i}}\left(2 d_{i}-z^{\prime}-z\right)}-\tilde{R}_{\mathrm{TM}}^{i, i-1} e^{-j k_{z_{i}}\left(2 d_{i}+z^{\prime}-z\right)}\right]\right) \\
& +\frac{k_{x} k_{z_{i}}}{k_{\rho}^{2}}\left(\tilde{R}_{\mathrm{TM}}^{i, i-1} M_{i}^{\mathrm{TM}}\right. \\
& \cdot\left[-e^{-j k_{z_{i}}\left(z+z^{\prime}\right)}+\tilde{R}_{\mathrm{TM}}^{i, i+1} e^{-j k_{z_{i}}\left(2 d_{i}+z-z^{\prime}\right)}\right] \\
& -\tilde{R}_{\mathrm{TE}}^{i, i-1} M_{i}^{\mathrm{TE}} \\
& \left.\left.\cdot\left[e^{-j k_{z_{i}}\left(z+z^{\prime}\right)}+\tilde{R}_{\mathrm{TE}}^{i, i+1} e^{-j k_{z_{i}}\left(2 d_{i}+z-z^{\prime}\right)}\right]\right)\right\}
\end{aligned}
$$


Then, rearranging the terms results in

$$
\begin{aligned}
\tilde{G}_{z x}^{A}= & -j k_{x} \frac{\mu_{i}}{2 j k_{z_{i}}} \frac{-j k_{z_{i}}}{k_{\rho}^{2}}\left\{e^{-j k_{z_{i}}\left(2 d_{i}-z^{\prime}-z\right)}\right. \\
& \cdot\left(\tilde{R}_{\mathrm{TE}}^{i, i+1} M_{i}^{\mathrm{TE}}+\tilde{R}_{\mathrm{TM}}^{i, i+1} M_{i}^{\mathrm{TM}}\right) \\
& +e^{-j k_{z_{i}}\left(2 d_{i}+z^{\prime}-z\right)} \\
& \cdot\left(\tilde{R}_{\mathrm{TE}}^{i, i+1} \tilde{R}_{\mathrm{TE}}^{i, i-1} M_{i}^{\mathrm{TE}}-\tilde{R}_{\mathrm{TM}}^{i, i+1} \tilde{R}_{\mathrm{TM}}^{i, i-1} M_{i}^{\mathrm{TM}}\right) \\
& +e^{-j k_{z_{i}}\left(2 d_{i}+z-z^{\prime}\right)} \\
& \cdot\left(\tilde{R}_{\mathrm{TM}}^{i, i-1} \tilde{R}_{\mathrm{TM}}^{i, i+1} M_{i}^{\mathrm{TM}}-\tilde{R}_{\mathrm{TE}}^{i, i-1} \tilde{R}_{\mathrm{TE}}^{i, i+1} M_{i}^{\mathrm{TE}}\right) \\
& +e^{-j k_{z_{i}}\left(z^{\prime}+z\right)} \\
& \left.\cdot\left(-\tilde{R}_{\mathrm{TE}}^{i, i-1} M_{i}^{\mathrm{TE}}-\tilde{R}_{\mathrm{TM}}^{i, i-1} M_{i}^{\mathrm{TM}}\right)\right\} .
\end{aligned}
$$

\section{The Green's Function $\tilde{G}_{x}^{q}$}

$$
\begin{aligned}
\tilde{G}_{x}^{q}= & \frac{1}{2 j \epsilon_{i} k_{z_{i}}}\left[e^{-j k_{z_{i}}\left|z-z^{\prime}\right|}+\frac{k_{z_{i}}^{2} B_{h}^{e}+k_{i}^{2} A_{h}^{e}}{k_{\rho}^{2}} e^{j k_{z_{i}}\left(z-z^{\prime}\right)}\right. \\
& \left.+\frac{k_{i}^{2} C_{h}^{e}-k_{z_{i}}^{2} D_{h}^{e}}{k_{\rho}^{2}} e^{-j k_{z_{i}}\left(z-z^{\prime}\right)}\right] .
\end{aligned}
$$

Substituting (39)-(42) into (45), the following expression is obtained:

$$
\begin{aligned}
\tilde{G}_{x}^{q}= & \frac{1}{2 j \epsilon_{i} k_{z_{i}}}\left\{e^{-j k_{z_{i}}\left|z-z^{\prime}\right|}+\frac{k_{z_{i}}^{2}}{k_{\rho}^{2}} \tilde{R}_{\mathrm{TM}}^{i, i+1} M_{i}^{\mathrm{TM}}\right. \\
& \cdot\left[e^{-j k_{z_{i}}\left(2 d_{i}-z^{\prime}-z\right)}-\tilde{R}_{\mathrm{TM}}^{i, i-1} e^{-j k_{z_{i}}\left(2 d_{i}+z^{\prime}-z\right)}\right] \\
& +\frac{k_{i}^{2}}{k_{\rho}^{2}} \tilde{R}_{\mathrm{TE}}^{i, i+1} M_{i}^{\mathrm{TE}} \\
& \cdot\left[e^{-j k_{z_{i}}\left(2 d_{i}-z^{\prime}-z\right)}+\tilde{R}_{\mathrm{TE}}^{i, i-1} e^{-j k_{z_{i}}\left(2 d_{i}+z^{\prime}-z\right)}\right] \\
& +\frac{k_{i}^{2}}{k_{\rho}^{2}} \tilde{R}_{\mathrm{TE}}^{i, i-1} M_{i}^{\mathrm{TE}} \\
& \cdot\left[e^{-j k_{z_{i}} t\left(z+z^{\prime}\right)}+\tilde{R}_{\mathrm{TE}}^{i, i+1} e^{-j k_{z_{i}}\left(2 d_{i}+z-z^{\prime}\right)}\right] \\
& -\frac{k_{z_{i}}^{2}}{k_{\rho}^{2}} \tilde{R}_{\mathrm{TM}}^{i, i-1} M_{i}^{\mathrm{TM}} \\
& \left.\cdot\left[-e^{-j k_{z_{i}}\left(z+z^{\prime}\right)}+\tilde{R}_{\mathrm{TM}}^{i, i+1} e^{-j k_{z_{i}}\left(2 d_{i}+z-z^{\prime}\right)}\right]\right\} .
\end{aligned}
$$

Then, rearranging the terms results in

$$
\begin{aligned}
\tilde{G}_{x}^{q}= & \frac{1}{2 j \epsilon_{i} k_{z_{i}}}\left\{e^{-j k_{z_{i}}\left|z-z^{\prime}\right|}+e^{-j k_{z_{i}}\left(2 d_{i}-z^{\prime}-z\right)}\right. \\
& \cdot\left(\frac{k_{z_{i}}^{2}}{k_{\rho}^{2}} \tilde{R}_{\mathrm{TM}}^{i, i+1} M_{i}^{\mathrm{TM}}+\frac{k_{i}^{2}}{k_{\rho}^{2}} \tilde{R}_{\mathrm{TE}}^{i, i+1} M_{i}^{\mathrm{TE}}\right) \\
& +e^{-j k_{z_{i}}\left(2 d_{i}+z^{\prime}-z\right)} \\
& \cdot\left(-\frac{k_{z_{i}}^{2}}{k_{\rho}^{2}} \tilde{R}_{\mathrm{TM}}^{i, i+1} \tilde{R}_{\mathrm{TM}}^{i, i-1} M_{i}^{\mathrm{TM}}\right. \\
& \left.+\frac{k_{i}^{2}}{k_{\rho}^{2}} \tilde{R}_{\mathrm{TE}}^{i, i+1} \tilde{R}_{\mathrm{TE}}^{i, i-1} M_{i}^{\mathrm{TE}}\right)+e^{-j k_{z_{i}}\left(2 d_{i}+z-z^{\prime}\right)} \\
& \cdot\left(-\frac{k_{z_{i}}^{2}}{k_{\rho}^{2}} \tilde{R}_{\mathrm{TM}}^{i, i+1} \tilde{R}_{\mathrm{TM}}^{i, i-1} M_{i}^{\mathrm{TM}}\right. \\
& \left.+\frac{k_{i}^{2}}{k_{\rho}^{2}} \tilde{R}_{\mathrm{TE}}^{i, i-1} \tilde{R}_{\mathrm{TE}}^{i, i+1} M_{i}^{\mathrm{TE}}\right)+e^{-j k_{z_{i}}\left(z^{\prime}+z\right)} \\
& \left.\cdot\left(\frac{k_{z_{i}}^{2}}{k_{\rho}^{2}} \tilde{R}_{\mathrm{TM}}^{i, i-1} M_{i}^{\mathrm{TM}}+\frac{k_{i}^{2}}{k_{\rho}^{2}} \tilde{R}_{\mathrm{TE}}^{i, i-1} M_{i}^{\mathrm{TE}}\right)\right\} .
\end{aligned}
$$

\section{REFERENCES}

[1] R. F. Harrington, Field Computations by Moment Methods. New York: Macmillan, 1968

[2] J. Jin, The Finite Element Method in Electromagnetics. New York: Wiley, 1993

[3] K. Kunz and R. Luebber, The Finite Difference Time Domain Method for Electromagnetics. Boca Raton, FL: CRC Press, 1993.

[4] J. R. Mosig and F. E. Gardiol, "General integral equation formulation for microstrip antennas and scatterers," Proc. Inst. Elect. Eng., vol. 132, pt. H, pp. 424-432, Dec. 1985.

[5] J. R. Mosig, "Arbitrarily shaped microstrip structures and their analysis with a mixed potential integral equation," IEEE Trans. Microwave Theory Tech., vol. 36, pp. 314-323, Feb. 1988.

[6] W. C. Chew, Waves and Fields in Inhomogeneous Media. New York: Van Nostrand, 1990.

[7] G. Dural and M. I. Aksun, "Closed-form Green's functions for general sources and stratified media," IEEE Trans. Microwave Theory Tech., vol. 43, pp. 1545-1552, July 1995

[8] Y. L. Chow, J. J. Yang, D. G. Fang, and G. E. Howard, "A closed-form spatial Green's function for the thick microstrip substrate," IEEE Trans. Microwave Theory Tech., vol. 39, pp. 588-592, Mar. 1991.

[9] S. L. Marple, Digital Spectral Analysis With Applications. Englewood Cliffs, NJ: Prentice-Hall, 1987.

[10] Y. Hua and T. K. Sarkar, "Generalized pencil-of-function method for extracting poles of an EM system from its transient response," IEEE Trans. Antennas Propagat., vol. 37, pp. 229-234, Feb. 1989.

[11] V. K. Jain, T. K. Sarkar, and D. D. Weiner, "Rational modeling by pencil-of-function method," IEEE Trans. Acoust., Speech, Signal Processing, vol. ASSP-31, pp. 564-573, June 1983.

[12] M. I. Aksun and R. Mittra, "Derivation of closed-form Green's functions for a general microstrip geometry," IEEE Trans. Microwave Theory Tech., vol. 40, pp. 2055-2062, Nov. 1992.

[13] M. I. Aksun, "A robust approach for the derivation of closed-form Green's functions," IEEE Trans. Microwave Theory Tech., vol. 44, pp. 651-658, May 1996

[14] M. I. Aksun and R. Mittra, "Estimation of spurious radiation from microstrip etches using closed-form Green's functions," IEEE Trans. Microwave Theory Tech., vol. 40, pp. 2063-2069, Nov. 1992.

[15] __ "Spurious radiation from microstrip interconnects," IEEE Trans. Electromag. Compat., vol. 35, pp. 148-158, May 1993.

[16] I. Park, R. Mittra, and M. I. Aksun, "Numerically efficient analysis of planar microstrip configurations using closed-form Green's functions," IEEE Trans. Microwave Theory Tech., vol. 43, pp. 394-400, Feb. 1995.

[17] K. Naishadham and T. W. Nuteson, "Efficient analysis of passive microstrip elements in MMIC's," Int. J. MIMICAE, vol. 4, no. 8, pp. 219-229, July 1994.

[18] L. Alatan, M. I. Aksun, K. Mahadevan, and T. Birand, "Analytical evaluation of the MoM matrix elements," IEEE Trans. Microwave Theory Tech., vol. 44, pp. 519-525, Apr. 1996.

[19] K. A. Michalski and J. R. Mosig, "Discrete complex image MPIE analysis of coax-fed coupled vertical monopoles in grounded dielectric substrate: Two formulations," IEE Proc. Microwave Antennas Propagat., vol. 142, pp. 269-274, June 1995.

[20] _ "Discrete complex image mixed-potential integral equation analysis of microstrip patch antennas with vertical probe feeds," Electromagnetics, vol. 15, pp. 377-392, July/Aug. 1995.

[21] K. A. Michalski and D. Zheng, "Electromagnetic scattering and radiation by surfaces of arbitrary shape in layered media, Part I: Theory," IEEE Trans. Antennas Propagat., vol. 38, pp. 335-344, Mar. 1990.

[22] A. Sommerfeld, Partial Differential Equations in Physics. New York: Academic, 1949.

[23] N. Kınayman and M. I. Aksun, "Comparative study of acceleration techniques for integrals and series in electromagnatic problems," Radio Sci., vol. 30, pp. 1713-1722, Nov./Dec. 1995.

[24] Sonnet Software, Inc., Em User's Manual, Liverpool, NY, 1995.

[25] J. M. Carroll and K. Chang, "Full-wave convergence analysis of microstrip transmission parameters," Int. J. MIMICAE, vol. 4, pp. 140-147, Apr. 1994

[26] W. F. Richards, Y. T. Lo, and D. Harrison, "An improved theory for microstrip antennas and applications," IEEE Trans. Antennas Propagat. vol. AP-29, pp. 38-46, 1981.

[27] W. F. Richards and Y. T. Lo, "Theoretical and experimental investigation of a microstrip radiator with multiple lumped linear loads," Electromagnetics, vol. 3, pp. 371-387, 1983.

[28] D. H. Schaubert, F. G. Farrar, A. Sindoris, and S. T. Hayes, "Microstrip antennas with frequency agility and polarization diversity," IEEE Trans. Antennas Propagat., vol. AP-29, pp. 118-123, Jan. 1981. 
Noyan Kinayman (M'96) was born in Ankara, Turkey, on November 22, 1968. He received the B.Sc. and M.Sc. degrees, both in electrical and electronic engineering, from Middle East Technical University (METU), Ankara, Turkey, in 1990 and 1993, respectively.

From 1990 to 1994, he worked in the Department of Preparation to Production, ASELSAN Military Electronics Inc., as a Design and Test Engineer. His major responsibility was to design computer-controlled and manual RF test systems which were used on the tests of various military and nonmilitary radios. In October 1994, he joined the Electrical Engineering Department, Bilkent University, Ankara, Turkey, as a Research Assistant His central professional interest is the numerical solutions of electromagnetic problems.
M. I. Aksun (M'92) received the B.S. and M.S. degrees, both in electrical and electronics engineering, from the Middle East Technical University, Ankara, Turkey, in 1981 and 1983, respectively, and the Ph.D. degree, in electrical and computer engineering, from the University of Illinois at Urbana-Champaign, in 1990.

From 1990 to 1992, he was a Post-Doctoral Fellow at the Electromagnetic Communication Laboratory, University of Illinois at Urbana-Champaign. Since 1992, he has been on the faculty of the Department of Electrical and Electronics Engineering, Bilkent University, Ankara, Turkey, where he is currently an Associate Professor. His research interests include the numerical methods for electromagnetics, microstrip antennas, and microwave and millimeter-wave integrated circuits. 